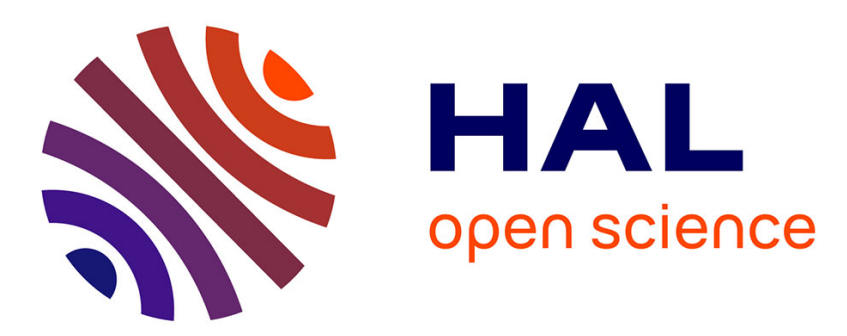

\title{
Digital implementation of the capacitively coupled electrical substitution for resistive bolometers
}

Matthieu Denoual, Sylvain Lebargy, Gilles Allègre

\section{To cite this version:}

Matthieu Denoual, Sylvain Lebargy, Gilles Allègre. Digital implementation of the capacitively coupled electrical substitution for resistive bolometers. Measurement Science and Technology, 2010, 21, pp.015205. 10.1088/0957-0233/21/1/015205 . hal-00979998

\section{HAL Id: hal-00979998 \\ https://hal.science/hal-00979998}

Submitted on 17 Apr 2014

HAL is a multi-disciplinary open access archive for the deposit and dissemination of scientific research documents, whether they are published or not. The documents may come from teaching and research institutions in France or abroad, or from public or private research centers.
L'archive ouverte pluridisciplinaire HAL, est destinée au dépôt et à la diffusion de documents scientifiques de niveau recherche, publiés ou non, émanant des établissements d'enseignement et de recherche français ou étrangers, des laboratoires publics ou privés. 


\title{
Digital implementation of the capacitively coupled electrical substitution for resistive bolometers
}

\author{
M Denoual, S Lebargy and G Allègre \\ GREYC-ENSICAEN, Université de Caen Basse Normandie, 6 bd maréchal Juin, 14000 Caen, France \\ E-mail: mdenoual@greyc.ensicaen.fr
}

\begin{abstract}
In this paper, digital implementation is proposed for the capacitively coupled electrical substitution for resistive bolometers. The capacitively coupled electrical substitution is a closed-loop configuration that allows operation of a resistive bolometer without any change in the structure of the sensor itself. The digital implementation of this closed-loop configuration linearizes the system composed of the bolometer and the electronics for the electrical substitution. Hence, a better optimization of the system control can be achieved. Besides, the use of the system and the set-up of the experiments are made easier with the digital implementation. The performance of the digital implementation was evaluated with optical radiation power stimulation and a resistive bolometer made on a glass membrane.
\end{abstract}

Keywords: uncooled bolometer, digital electrical substitution, thermal feedback

\section{Introduction}

Resistive bolometers are one type of infrared sensor $[1,2]$. Bolometers measure radiation energy or power, in the infrared range for example, by a measure of the temperature rise of an absorbing material exposed to the radiation. The relation between incoming radiation power and electrical signal depends on both laws of thermal exchange and laws of electricity. The thermal laws describing the three heat loss mechanisms (conduction, convection and radiation) determine the nonlinear response of the system depending on technology and temperature domain. Usually, in resistive bolometers' setup, operation in a moderate vacuum minimizes the convection losses, and a temperature-controlled screen can be used to limit the radiation losses. Then, conduction remains the predominant heat loss mechanism between the bolometer and its heat sink. This mechanism which is strongly nonlinear at low temperature still remains nonlinear at room temperature. The expression used for cryogenic bolometers is $K\left(T^{n}-T_{s}^{n}\right)$, where $T_{s}$ is the temperature of the heat sink, $K$ is a material and geometry-dependent parameter and $n$ is a number between 4 and 6 depending on the dominant thermal impedance [3].
For uncooled bolometers, the conduction heat loss expression is also nonlinear because of the temperature dependence of the thermal conductance $[4,5]$. For resistive bolometers, the electro-thermal effect due to the resistor biasing [1] also induces nonlinear behavior. In both cases the expression can be linearized when small fluctuations around a working temperature are considered and becomes $G_{\text {eff }}\left(T-T_{S}\right)$, where $G_{\text {eff }}$, the effective thermal conductance, is constant.

Nowadays, demand is for low-cost uncooled bolometers for infrared imaging devices [6]. Uncooled bolometers are more compact and easier to use than cooled or cryogenic ones, but their signal-to-noise ratio is poorer than cooled or cryogenic bolometers mainly because of noise dependence on temperature. Therefore, uncooled resistive bolometers have to be designed and fabricated so as to increase their intrinsic responsivity (equation (1)) to incoming radiation $[2,6]$ :

$$
R[V / W]=\frac{V_{o}}{P_{\text {radiation }}}=\frac{\beta \eta I_{\text {bias }} R_{\text {sensing }}}{\left(G_{\text {eff }}^{2}+\omega^{2} C_{\text {th }}^{2}\right)^{\frac{1}{2}}},
$$

with $V_{o}$ the output voltage, $P_{\text {radiation }}$ the power of the incoming radiation, $R_{\text {sensing }}$ the resistance of the sensing resistor, $\beta$ the temperature coefficient of $R_{\text {sensing }}, \eta$ the absorption coefficient 
of the absorbing layer, $I_{\text {bias }}$ the bias current and $C_{\mathrm{th}}$ the thermal capacitance. $G_{\text {eff }}$ is expressed to take into account the electrothermal effect as follows:

$$
G_{\text {eff }}=G_{\text {th }}-\left(\frac{\delta R_{\text {sensing }}}{\delta T}\right) I_{\text {bias }}^{2},
$$

with $G_{\text {th }}$ the conductance of the thermal link between the bolometer and the substrate.

In order to obtain the best intrinsic responsivity of a bolometer, one will choose the best materials based upon their availability and the compatibility of their fabrication processes: resistive material with the highest temperature coefficient of resistance (TCR) for the sensing resistor-VOx [2], high-temperature superconductors (YBCO) or manganese films (LCMO, LSMO) [7, 8]-absorbing material with the highest absorption coefficient $(\eta)$ at the infrared wavelength target range for the absorbing layer-black gold [9, 10], nanostructured materials [11] - structural material with the lowest thermal mass $C_{\mathrm{th}}$ and thermal conductance $G_{\mathrm{th}}-$ polymers, polyimide [12]. Once the materials are selected, one will also develop the best geometries or structures to optimize, i.e. usually reduce, $C_{\mathrm{th}}$ and $G_{\mathrm{th}}$. Miniaturization [13-16] and membrane structures $[15,16]$ are the two ways frequently explored.

For a given bolometer and considering its intrinsic performance, one can improve the performance of the system composed of the bolometer and the associated readout electronics thanks to the closed-loop configuration. The closed-loop configuration of bolometers, as for any physical system, reduces the time response of the system and the static error. A closed-loop configuration also improves the linearity and the range of the measurement and can reduce aging when materials such as polymers are considered because of limited temperature cycles. Closed-loop configurations using the Joule effect to feedback heat have been proposed [16-19]. These configurations use the electrical substitution (ES) principle that allows the feedback of heat, i.e. heat variations due to the radiation are compensated by heat produced by electrical means in practice thanks to a current flowing into a heating resistor.

The basic concept is to keep the temperature of the resistive sensor constant thanks to a feedback electrical Joule effect power that completes the incoming radiation power so that the total amount of power at the detector is kept constant (figure 1). Recently, we have proposed a new configuration, the capacitively coupled electrical substitution (CCES) [20]. The performance of this CCES configuration was demonstrated using an analog implementation. In contrast with previous configurations, the CCES is applicable to every existing bolometer without any design modification provided that we are not limited by the bandwidth of the electronic circuits commercially available for the realization of the feedback circuits. With the CCES configuration both the electrical and thermal bias points can be set independently. These specificities are enabled by the capacitive coupling basis of this new configuration.

This paper presents a digital implementation of this new CCES configuration. Two main advantages of the digital implementation are the ease of use and the linearization

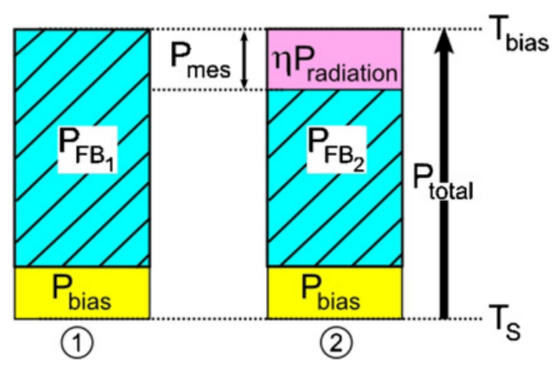

Figure 1. Electrical substitution principle used for heat feedback. (1) The feedback power, $P_{\mathrm{FB}}$, produced by the electrical Joule effect, in the absence of incoming radiation power defines the thermal bias point $T_{\text {biaspoint }}$. (2) When radiation power, $P_{\text {radiation }}$, is absorbed onto the bolometer, the feedback power $P_{\mathrm{FB}}$ evolves to keep the total amount of power, $P_{\text {total }}$, constant. $T_{s}$ is the sink ambient or thermostat temperature. $P_{\text {bias }}$ corresponds to the power dissipated in the sensing resistor due to bias current $\left(R_{\text {sensing. }} . I_{\text {bias }}^{2}\right) . P_{\text {total }}$ is balanced by $P_{\text {sink }}$ which represents power loss to the environment. For small differences between $T_{\text {bias }}$ and $T_{s}$ that allow assumption of a constant $G_{\text {th }}$ and therefore a constant $G_{\text {eff }}$, we can write the simplified and linearized equation $P_{\text {sink }}=G_{\text {eff }}\left(T_{\text {bias }}-T_{s}\right)$. The closed loop that ensures a constant $T_{\text {bias }}$ thus ensures $P_{\text {total }}$ is kept constant. $P_{\text {mes }}=P_{\text {total }}-P_{\text {bias }}-P_{\mathrm{FB}}$ is a measure of the radiation power absorbed by the bolometer and corresponds to the decrease of the feedback power in the presence of radiation power.

of the heat feedback. The experimental set-up was based on a resistive bolometer deposited on a glass membrane and radiation power stimulation from LEDs. The digital implementation of the CCES configuration was successfully demonstrated.

\section{Capacitively coupled electrical substitution and digital implementation}

\subsection{Global view of the implementation}

The system is composed of four main parts: the bolometer, the conditioning electronics, the control system and the capacitively coupled feedback and matching electronics (figure 2). Part 1 is the bolometer. The absorption layer converts incoming radiation into heat and which in turn changes the resistance of the temperature-sensing resistor. This sensing resistor is part of a modified Wheatstone bridge. Part 2 also contains the amplification and filtering electronics. Part 3 contains the control engineering for closed-loop system performance adjustments. Part 4 modulates the feedback control voltage and couples it to the sensing resistor of the bolometer.

Digital implementation applies to the interfacing between the conditioning electronics and the bolometer and to part 3 .

\subsection{Digital implementation linearizes the system}

Several advantages are expected from the digital implementation of the CCES configuration. The digital implementation implies an easy interfacing of the system for bias point setting and measure readout. Digital implementation is more versatile and allows easier adjustments of controller implementation for the closed-loop 


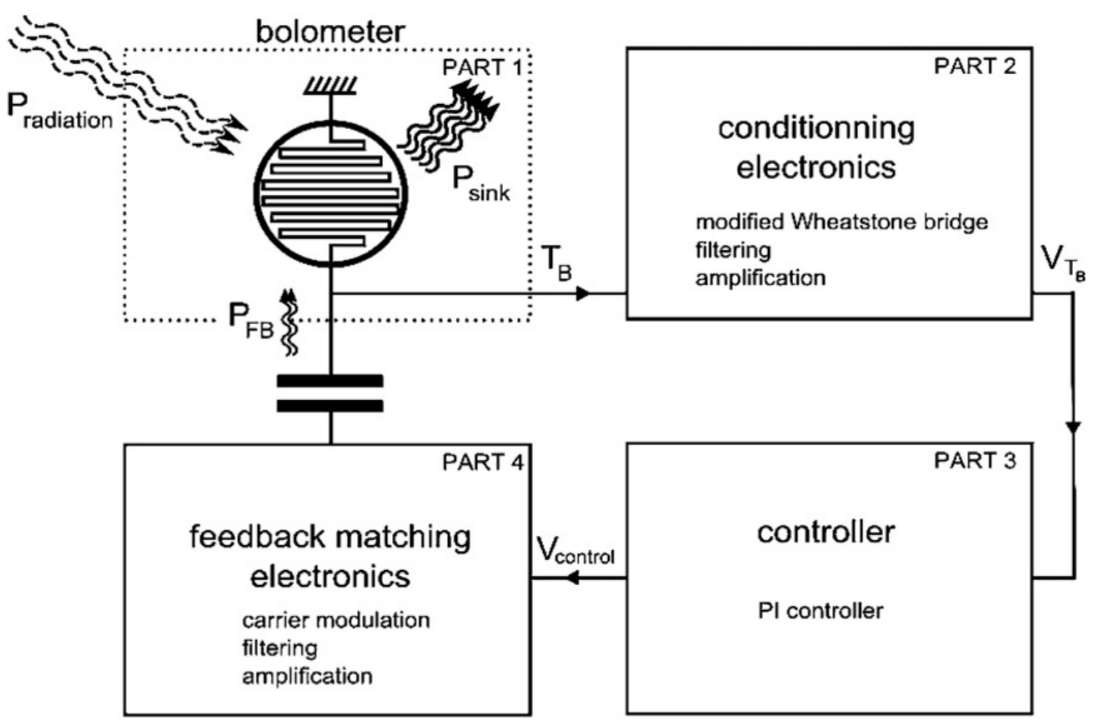

Figure 2. Scheme of the global view of the capacitively coupled electrical substitution implementation. The absorption layer of the bolometer converts the incoming radiation power variation into temperature variation. The conditioning electronics, part 2 , allows the measurement of this temperature variation. This measure compared to a reference value serves as an input for the controller (part 3) which evaluates the feedback control signal. This signal is matched with part 4 to be coupled to the sensing resistor.

configuration. Finally, the digital implementation and the use of pulse width modulation linearize the open-loop system response over the overall input range and therefore allow easier design of the feedback controller and provide linearity for the overall measurement range. The linearization is demonstrated through equations below.

The control signal $V_{\text {control }}$ comes from a pulse width modulation (PWM) output of the microcontroller. Through a switch, the control signal modulates a carrier whose frequency is much higher than the bolometer bandwidth (typically $1 \mathrm{MHz}$ with a constant amplitude $V_{\text {carrier }}$ ).

$$
\overline{V_{\text {control }}}=\alpha V_{\max },
$$

where $\alpha$ is the cyclic ratio of the PWM output of the microcontroller and $V_{\max }$ the constant output high-level voltage. Then the RMS voltage coupled to the sensing resistor can be expressed as

$$
\begin{aligned}
\tilde{V}_{\mathrm{FB}}^{2} & =\frac{1}{T} \int_{0}^{\alpha T} V_{\text {carrier }}^{2} \cdot \sin ^{2}\left(\omega_{\text {carrier }} t+\phi\right) \mathrm{d} t \\
& =\frac{V_{\text {carrier }}^{2}}{2} \cdot\left(\alpha-\frac{1}{T} \int_{0}^{\alpha T} \cos \left(2 \omega_{\text {carrier }} t+2 \phi\right) \mathrm{d} t\right),
\end{aligned}
$$

with $\Phi$ the phase of the carrier at the PWM modulation beginning. Defining $T_{\text {carrier }}$ as the period of the carrier and $T$ the period of the PWM modulation, we can express $T=$ $n T_{\text {carrier }}+\tau$, with $\tau<T_{\text {carrier }}$ and $n$ an integer. The RMS voltage coupled to the sensing resistor can be expressed as

$$
\tilde{V}_{\mathrm{FB}}^{2}=\frac{V_{\text {carrier }}^{2}}{2} \cdot\left(\alpha-\frac{\sin \left(2 \alpha \omega_{\text {carrier }} \tau+2 \phi\right)-\sin (2 \phi)}{2 n+2 \omega_{\text {carrier }} \tau}\right)
$$

assuming $n \gg 1$, the RMS voltage expression can be simplified and linearized:

$$
\tilde{V}_{\mathrm{FB}}^{2} \simeq \frac{V_{\text {carrier }}^{2}}{2} \cdot \alpha \text {. }
$$

The term depending on $n$ inducing a linearity error could be totally cancelled by synchronization between the carrier and PWM modulation, i.e. having $\tau$ equal to 0 . Hence,

$$
\tilde{V}_{\mathrm{FB}}^{2} \propto \overline{V_{\text {control }}} \text {. }
$$

Therefore, the heat feedback power, $P_{\mathrm{FB}}$, in the sensing resistor is proportional to the value of the feedback digital controller $V_{\mathrm{PI}}$ :

$$
P_{\mathrm{FB}}=\frac{\tilde{V}_{\mathrm{FB}}^{2}}{R_{\text {sensing }}} \propto \overline{V_{\text {control }}} \text {. }
$$

The linearization of the open-loop system ensures identical error, noise and controller performance over the whole operating range of the system. The linearization also enables a best optimization of the controller design.

Figure 3 illustrates the theoretical curves of the feedback power depending on the CCES configuration type. With an analog configuration, the gain in open loop varies as the slope of the parabolic curve. The design of the controller to ensure system stability will take into account the highest gain (right part of the curve, high values of $V_{\text {control, highest slope), and }}$ therefore poorer performance will be achieved for smaller gain values (left part of the curve, small values of $V_{\text {control }}$ ). In summary, in an analog configuration, the performance of the closed-loop system depends on the working point; in a digital configuration, the performance of the closed loop is identical over the whole range.

\section{Experiments}

Experiments with optical source were conducted to validate the digital implementation of the CCES configuration. 


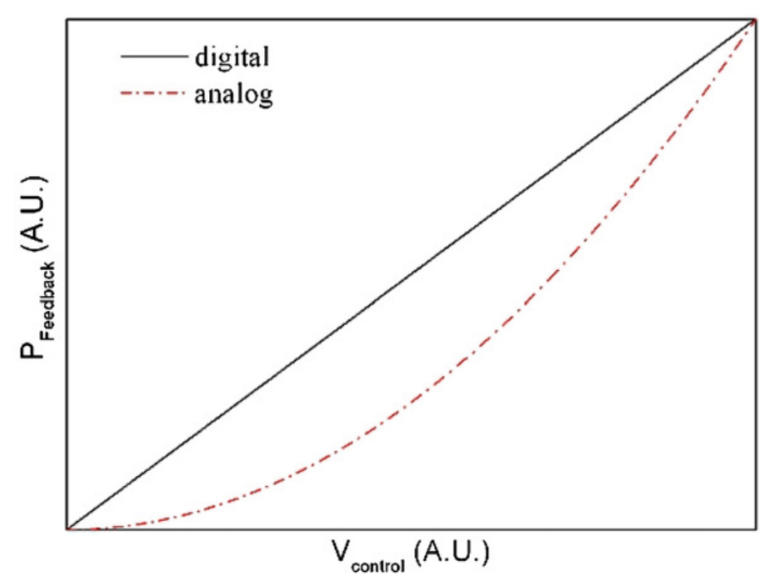

Figure 3. Theoretical curves giving the shape of the feedback power according to the CCES implementation type. Analog implementation produces a quadratic feedback power. In contrast, digital implementation gives a linear feedback power.

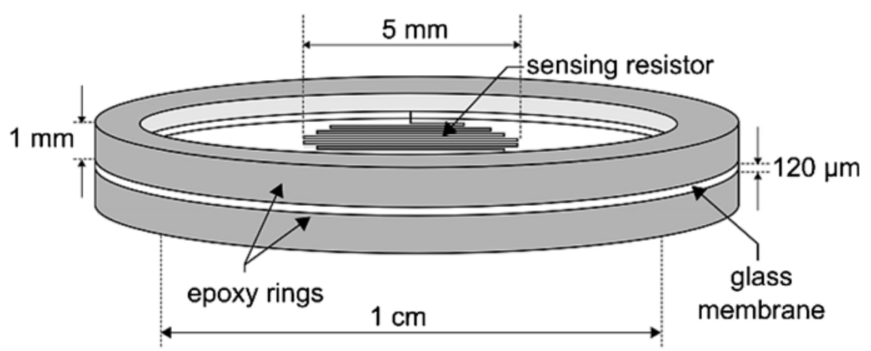

Figure 4. Sketch of the glass bolometer structure with sensing resistor on the top side and heating resistors at the back side.

\subsection{Description of the bolometer}

The bolometer chosen was described in [20] and is illustrated in figure 4. Practical considerations motivated this choice. This bolometer is robust and easy to realize. The drawback is poor intrinsic performance: $\tau_{\text {th }}=11.7 \mathrm{~s}$ with $C_{\text {th }}=$ $14.6 \mathrm{~mJ} \mathrm{~K}^{-1}$ and $G_{\text {eff }}=1.25 \mathrm{~mW} \mathrm{~K}^{-1}$. These parameters are extracted assuming a first-order model for the bolometer. This bolometer consists of three gold resistors placed on either side of a $120 \mu \mathrm{m}$ thick glass membrane. On the top side, one gold resistor, $R_{\text {sensing }}(80.6 \Omega)$, represents the temperature-sensing resistor with a temperature coefficient $\beta=9.2 \times 10^{-4} \mathrm{~K}^{-1}$. On the backside, two gold resistors, $R_{\text {heating1 }}$ and $R_{\text {heating2 }}$, can be used for membrane heating. These heating resistors were previously used for heat feedback in the conventional electrical substitution configuration and for performance characterization with a standard procedure.

\subsection{Digital implementation of the CCES configuration}

The digital part of the system was implemented in a microcontroller (dsPIC30F2010) (figure 5). This microcontroller digitizes the output voltage of the analog conditioning electronics $V_{\mathrm{TB}}$ with a 16 bit $\mathrm{ADC}$ and $100 \mathrm{~ms}$ sampling time. A proportional, integral (PI) algorithm is implemented. The voltage control, $V_{\text {control, }}$ is calculated and comes out of the DSPIC microcontroller as a pulsed-widthmodulated signal. Digitized $V_{\mathrm{TB}}$ and calculated $V_{\text {control }}$ are displayed on a PC screen via an USB connection and a Labview interface. The Labview interface with the microcontroller drives the independent setting of the electrical bias point and the thermal bias point. Two DACs connected to the modified Wheatstone bridge set the electrical bias point. Constant power applied using the controller part in the microcontroller sets the thermal bias point.

\subsection{Experimental setup}

The electrical bias set-up is set by the DACs. One DAC is dedicated to bias each of the modified Wheatstone half bridges. The control voltage modulates the carrier by means of a switch.

The feedback signal $\tilde{V}_{\mathrm{FB}}$ is supplied to the sensing resistor through a capacitor to provide the power feedback $P_{\mathrm{FB}}$. This capacitive coupling provides for invariance of the electrical bias point of the sensing resistor and consequently the sensitivity of the temperature measurement [20].

A digital PI controller is implemented to zero the error and to allow direct reading of the power absorbed onto the bolometer. All the measurements are performed at room temperature in a vacuum chamber under primary vacuum ( 2 mTorr) to limit convection heat loss (by assuming an emissivity of 1.0, the radiation loss was estimated to be $10 \%$ of the conduction loss). The carrier was produced by a quartz oscillator with a $1 \mathrm{MHz}$ frequency. The PWM frequency of the microcontroller output was set at $8 \mathrm{kHz}$ for all the experiments.

3.3.1. Measurement procedure. Firstly, the system is turned on in the open-loop configuration, the vacuum pumping is started and no optical stimulus is applied. Secondly, the electrical bias point is set with the DACs, to balance the modified Wheatstone bridge. A constant cyclic ratio for $V_{\text {control }}$ defines the power applied to the sensing resistor and hence the thermal bias point. Once thermalized, the system is switched to the closed-loop mode. At this point, the optical power can be applied for measurement.

3.3.2. Optical power excitation. The system was characterized through optical tests. Optical power was delivered by LEDs. A double modulation of the power supply of the LEDs allowed optical power intensity and frequency variations with square modulation (figure 6). Firstly a highfrequency pulsed-width modulation, with frequency out of the bandwidth of the bolometer system, allows defining the mean optical radiation power with the cyclic ratio of the pulses. Secondly, a low-frequency modulation, in the bolometer system bandwidth, allows square modulation (on-off).

3.3.3. Calibration. The optical power absorbed on the bolometer surface was estimated by comparison of the resistive bolometer response to an equivalent electrical Joule effect power produced by a heating resistor placed on the backside of the bolometer glass membrane. Following the same standard 


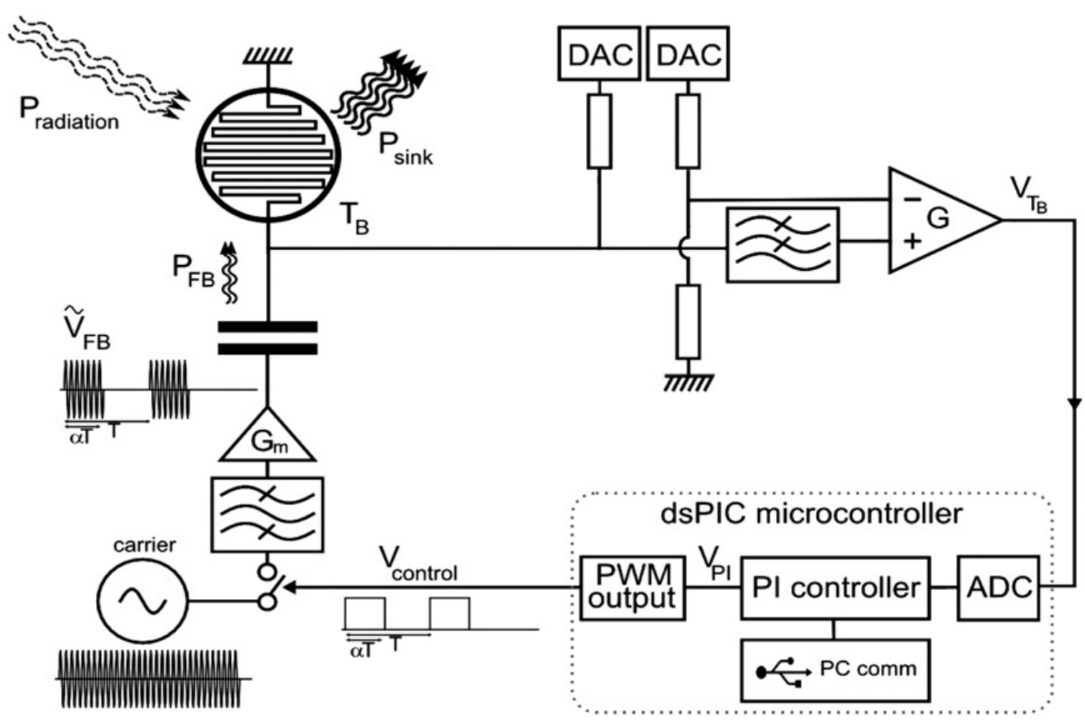

Figure 5. Electrical schematic of the system.

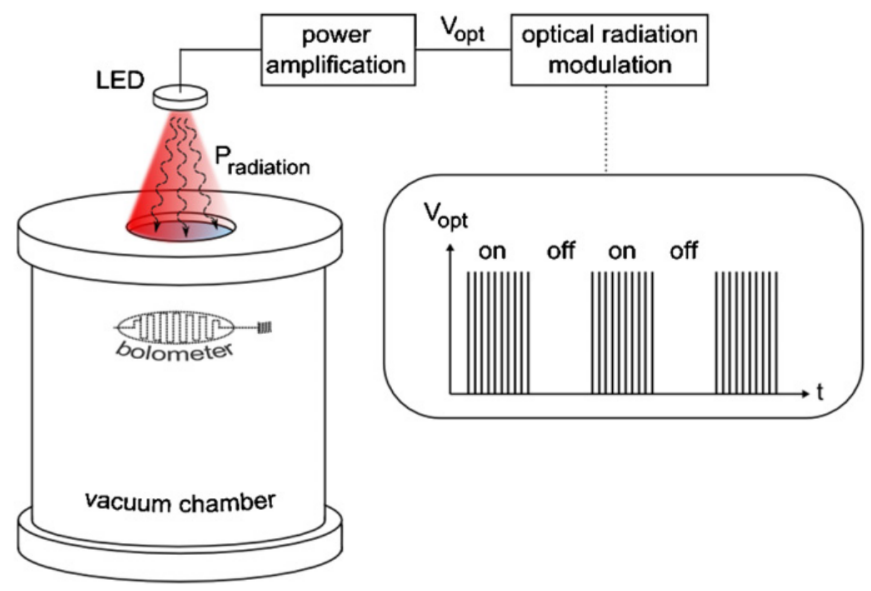

Figure 6. Set-up for experiments. The optical radiation source illuminates the bolometer placed in a vacuum chamber through a porthole.

procedure as described in [20], voltage square pulses are delivered through the $4.5 \mathrm{k} \Omega$ backside heating resistor to produce known Joule heat stimuli. Comparison between the response to Joule stimuli and optical power stimuli reveals that a $50 \%$ optical power cyclic ratio modulation corresponds to $350 \mu \mathrm{W}$ electrical stimulation.

\section{Results}

Figure 7 illustrates the typical response of the system in the open-loop and closed-loop configurations. In the openloop configuration, at the beginning of the recording, the temperature of the sensing resistor varies according to the incoming optical radiation power fluctuations and the constant feedback power applied sets the thermal bias point. In the closed-loop configuration, the feedback power is adjusted by the controller in order to compensate the incoming optical radiation so as to keep the temperature of the sensing resistor constant. As expected the time response of the system in the closed-loop configuration $\left(t_{5 \%}<3 \mathrm{~s}\right)$ is smaller than in the open-loop configuration $\left(\tau_{\text {th }}=11.7\right.$ s, i.e. $t_{5 \%}=3 \tau_{\text {th }}=$ $35.1 \mathrm{~s})$.

In this example, in the open-loop configuration the system is not fast enough to reach its final value for a $10 \mathrm{mHz}$ input optical power stimulus. The closed-loop configuration produces a reduced time drift compared to the open-loop configuration since the temperature at the resistive bolometer location is kept constant. The remaining drift is due to the overall vacuum chamber temperature evolution that serves as thermostat here.

Figure 8 illustrates the linear proportionality between the incoming optical radiation power, fixed by the duty cycle, and the feedback power $P_{\mathrm{FB}}$. In the electrical substitution configuration, $P_{\mathrm{FB}}$ is the output measurement value which produces easy power readings of the radiation power received and absorbed on the bolometer surface through its variations. The $P_{\text {mes }}$ values for each point were obtained by fast Fourier transform analysis of the feedback power signal recorded for ten periods of the incoming optical radiation. The linearity error is less than $0.8 \%$. Several error sources may contribute to this linearity error: model approximation depending on $n$ introduced in section 2.2, the signal quantization, digital calculus approximation and obviously the signal-to-noise ratio of the system. The total amount of power on the membrane of the bolometer is shown to be constant within this linearity error.

Figure 9 represents the power spectral densities for measurements performed in both open-loop and closed-loop configurations. The reshaping of the noise power spectral density due to the control feedback [20] in the closed-loop configuration can be observed. The figure also shows that there is no degradation of the signal-to-noise ratio when the bolometer is operated in the closed-loop configuration, it is rather enhanced because of the reduction of the low-frequency drifts. 
Table 1. Time response for various configurations.

\begin{tabular}{llll}
\hline & Open-loop & \multicolumn{2}{c}{ Closed-loop } \\
\hline Configuration & & Analog CCES & Digital CCES \\
Time response $t_{5 \%}$ & $35.1 \mathrm{~s}$ & $2.4 \mathrm{~s}(3 \times 0.8 \mathrm{~s} \mathrm{[20])}$ & $<3 \mathrm{~s}$ \\
\hline
\end{tabular}

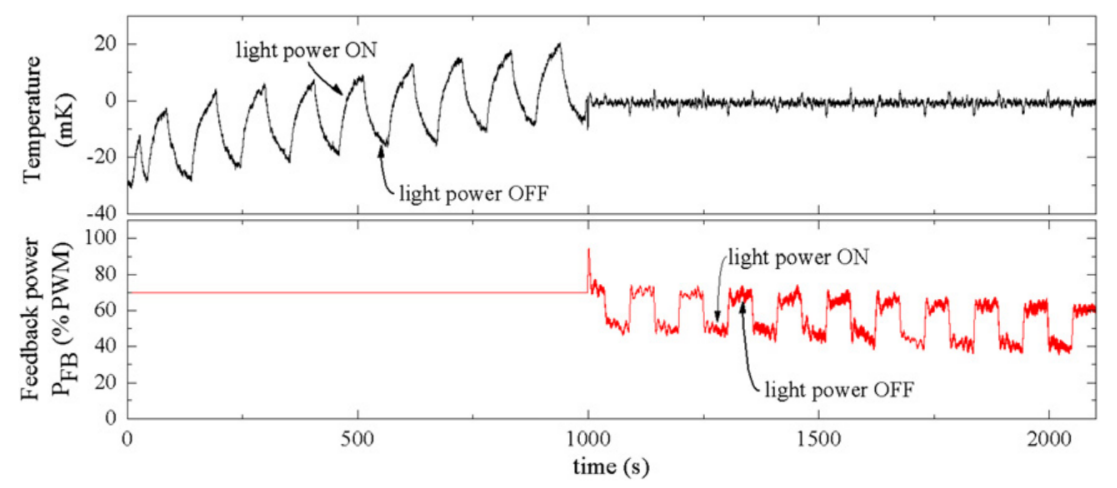

Figure 7. Open-loop ( $t=0 \mathrm{~s}$ to $t=1000 \mathrm{~s}$ ) followed by closed-loop (from $t=1000 \mathrm{~s}$ ) response of the bolometer with the digital implementation of the capacitively coupled electrical substitution. $10 \mathrm{mHz}, 50 \%$ incident light power. Temperature of the resistive sensor of the bolometer (top) and feedback power when the system is in the closed-loop configuration (bottom). The thermal bias point was set in this case for $70 \%$ of the maximum power feedback. The temperature zero is defined for the thermal bias point.

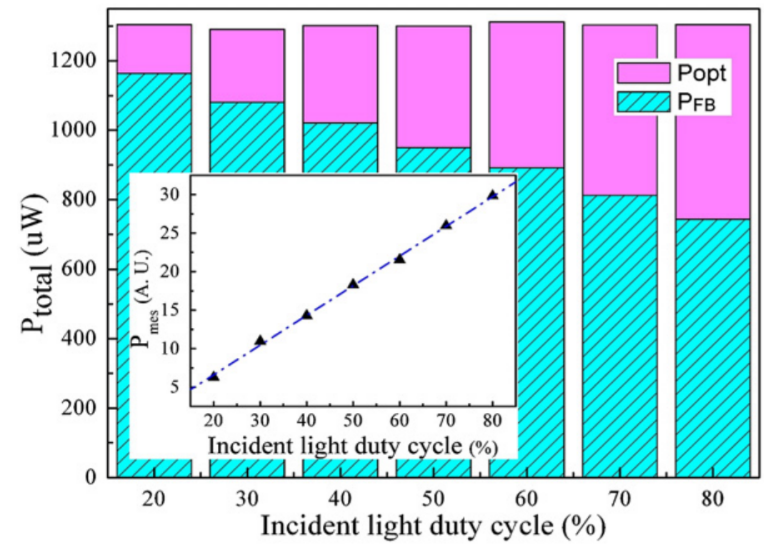

Figure 8. The main back graph represents the total power applied to the bolometer, with the two main components: the optical radiation power $\left(P_{\mathrm{opt}}\right)$ and the resulting feedback power $\left(P_{\mathrm{FB}}\right)$. The insert represents the measured received power $P_{\text {mes }}$ versus incident radiation power with linear regression (dotted line). For these measurements, the frequency of the optical radiation power was $30 \mathrm{mHz}$ and the light duty cycle allowed to control the mean optical power delivered as explained in the optical power excitation subsection. The thermal working point was set at $70 \%$ of the maximum feedback power. $P_{\text {mes }}$ was extracted from analysis over ten periods of the optical power radiation.

\section{Discussion}

The results illustrate the performance of a digital implementation of the capacitively coupled electrical substitution configuration. The digital CCES implementation, like the analog implementation studied previously [20], allows taking advantage of the operation in the closed-loop configuration without any extra heating element. Therefore, for a given bolometer, the operation in the closed-loop

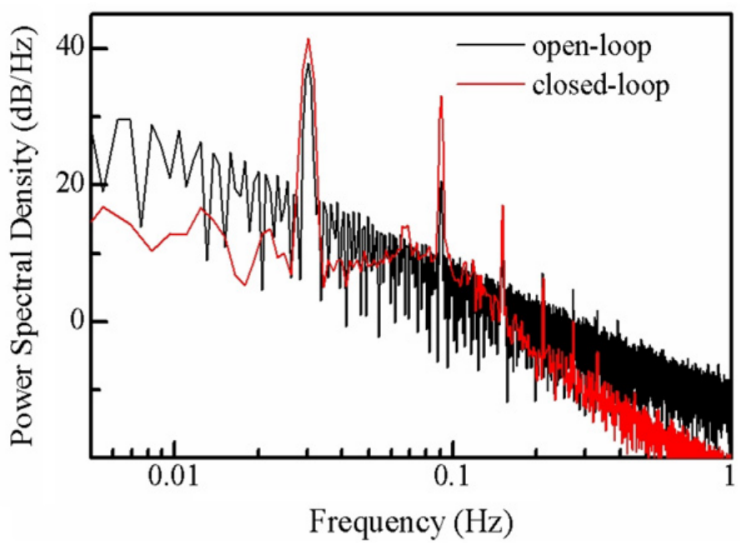

Figure 9. Power spectral densities in both open-loop and closed-loop cases. A $30 \mathrm{mHz} 50 \%$ light power, optical power stimulus was applied during the recording.

configuration allows for time response reduction while the signal-to-noise ratio remains the same.

Compared to the analog implementation, and as expected from theory, the performance in terms of time constant reduction (table 1) and signal-to-noise ratio are the same. Indeed, the type of implementation has no impact on those features provided that the quantification noise in the case of the digital implementation is lower than the equivalent noise of the bolometer and conditioning electronics.

In the closed-loop configuration, either analog or digital, the PI controller zeroes the error between the bias temperature set point and the temperature of the bolometer, improving the stability of the temperature of the bolometer. Aging of sensing resistors can be reduced in this configuration since thermal cycles are reduced. Furthermore, this can allow the use of material more fragile to thermal cycles for the fabrication of 
the bolometer, especially, polymer material for membranetype bolometers.

The two main differences between the analog and digital implementation concern the linearity and the usability. Firstly, as demonstrated in the paper, the digital implementation linearizes the heat feedback and therefore the whole open-loop system allowing simpler and more efficient design of feedback controller. This characteristic has not been illustrated here because of the poor intrinsic performance of the bolometer used; the illustration of this potential optimization will be part of future work involving improved bolometers. Secondly, the digital implementation makes it easier to set the working points. The control of the thermal working point is especially interesting for calibration and self-testing. The possibility of controlling the working point will also be interesting if one uses bolometers made of sharp resistive transition materialsfor example high- $T_{c}$ materials such as LSMO.

As for accelerometers for many years now, the digital implementation of the feedback loop with bolometers will allow for new functionalities such as self-testing, selfcalibration and dynamic change of the measurement range opening the doors to smart bolometers.

\section{Conclusion}

Digital implementation of the capacitively coupled electrical substitution configuration for resistive bolometers has been demonstrated. The feedback PI control was implemented in a microcontroller and associated with a bolometer with conditioning and matching electronics. The digitized system operated successfully in the closed-loop configuration when illuminated by an optical radiation source. A linearity better than $1 \%$ was observed between applied optical power and the measured received power. The digital implementation makes the system setting easier and opens interesting perspectives in term of self-calibration and self-testing.

\section{References}

[1] Richards P L 1994 Bolometers for infrared and millimeter waves J. Appl. Phys. 76 1-24

[2] Kruse P W 2001 Uncooled Thermal Imaging: Arrays, Systems and Applications (Bellingham, WA: SPIE Optical Engineering Press)
[3] Irwin K D 1995 An application of electrothermal feedback for high resolution cryogenic particle detection Appl. Phys. Lett. 66 1998-2000

[4] Sze S 1981 Physics of Semiconductor Devices 2nd edn (New York: Wiley)

[5] Ferro-Ceramic Grinding Inc 2000 Ceramic materials physical, mechanical, thermal and electrical properties reference chart http://ferroceramic.com/

[6] Fièque $\mathrm{B}$, Tissot $\mathrm{J} \mathrm{L}$, Trouilleau $\mathrm{C}$, Crastes $\mathrm{A}$ and Legras $\mathrm{O}$ 2007 Uncooled microbolometer detector: recent developments at Ulis Infrared Phys. Technol. 49 187-91

[7] Hao J H, Mao X, Chen C H and Lu D X 1999 Room temperature bolometric applications using manganese oxide thin films Int. J. Infrared Millim. Waves 20 2113-20

[8] Zerov V Y and Malyarov V G 2001 Heat-sensitive materials for uncooled microbolometer arrays J. Opt. Technol. 68939

[9] Sintsov V N 1966 A study of the properties of gold black J. Appl. Spectrosc. 4 362-5

[10] Becker W, Fettig R, Gaymann A and Ruppel W 1996 Black gold deposit as absorbers for far infrared radiation Phys. Status Solidi b 194 241-55

[11] Kokabi A, Moftakharzadeh A, Ghods-Elahi T, Vesaghi M, Schubert J and Fardmanesh M 2008 Effect of the nano-structure $\mathrm{Cu}$-carbon composite absorber layer on the response of superconductive YBCO TEBs J. Phys.: Conf. Ser. 129012044

[12] Dayed S A, Butler D P and Celik-Butler Z 2005 Micromachined infrared bolometers on flexible polyimide substrates Sensors Actuators A 118 49-59

[13] Hwang T L, Schwarz S E and Rutledge D B 1979 Microbolometers for infrared detection Appl. Phys. Lett. $34773-6$

[14] Atlass M 1992 Microstructure design for high IR sensitivity Patent WO 94/01743

[15] Mahmood A, Butler D P and Celik-Butler Z 2006 Micromachined bolometers on polyimide Sensors Actuators A 132 452-9

[16] Allègre G, Guillet B, Robbes D, Méchin L, Lebargy S and Nicoletti S 2007 A room temperature $\mathrm{Si}_{3} \mathrm{~N}_{4} / \mathrm{SiO}_{2}$ membrane-type electrical substitution radiometer using thin film platinum thermometers Meas. Sci. Technol. 18 183-9

[17] Rice J P, Lorentz S R, Datla R U, Vale L R, Rudman D A, Sing M L C and Robbes D 1998 Active cavity absolute radiometer based on high- $T_{\mathrm{c}}$ superconductors Metrologia 35289

[18] Rice J P 2000 An electrically substituted bolometer as a transfer-standard detector Metrologia 37 433-6

[19] Oliveira A, Palma L S, Costa A S, Freire R C S and Lima A C de C 2006 A constant temperature operation thermoresistive sigma-delta solar radiometer Measurement 39 267-73

[20] Denoual M, Delaunay S, Allègre G and Robbes D 2009 Capacitively coupled electrical substitution for resistive bolometer enhancement Meas. Sci. Technol. 20015105 